\title{
Characterization of Superelastic NiTi Alloys by Nanoindentation: Experiments and Simulations
}

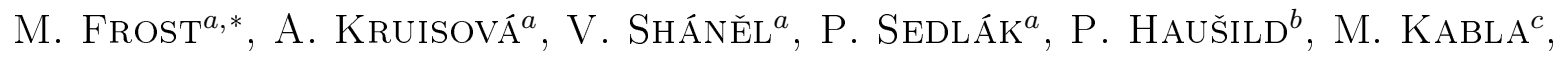 \\ D. $\mathrm{SHILO}^{c}$ AND M. LANDA ${ }^{a}$ \\ ${ }^{a}$ Institute of Thermomechanics, Academy of Sciences of the Czech Republic, \\ Dolejškova 5, Praha 18000, Czech Republic \\ ${ }^{b}$ Faculty of Nuclear Sciences and Physical Engineering, Czech Technical University, \\ Trojanova 13, Praha 12000, Czech Republic \\ ${ }^{c}$ Faculty of Mechanical Engineering, Technion - Israel Institute of Technology, \\ Haifa 32000, Israel
}

\begin{abstract}
NiTi-based shape memory alloys are metallic materials exhibiting remarkable response to mechanical and/or thermal loading, e.g. superelasticity, pseudoplasticity or one-way shape memory effect. They can be engineered into structures of micro-size dimensions, hence, they appear promising for application in micro-electromechanical systems. For their efficient utilization, appropriate characterization is important. Due to relative simplicity, indentation represents a very standard and popular technique for basic characterization of metallic materials providing information about stiffness and hardness. Moreover, it can be used for identification of other events in the material. This study aims to verify applicability of the recently developed constitutive model for NiTi-based shape memory alloy in simulations of nanoindentation tests. The model is fitted to a particular sample material using experimental data, and a series of simulations mimicking tests at various temperatures is performed. Since the model also captures two-stage martensitic transformation via the intermediate $R$-phase, its influence on the simulations is investigated as well. It is confirmed that spherical indentation is a suitable method for simple and fast detection whether the material is in superelastic or pseudoplastic regime.
\end{abstract}

DOI: 10.12693 /APhysPolA.128.664

PACS: $62.20 . \mathrm{fg}, 46.15 .-\mathrm{x}$

\section{Introduction}

Instrumented indentation has been widely applied to characterize the mechanical behavior of materials at small scale. The well-known example is the Oliver-Pharr method $[1,2]$ frequently adopted to determine elastic (indentation) modulus of elasto-plastic materials, basically from a couple of characteristics of the measured indentation response and known material and geometrical properties of the indenter. However, the shape of the loaddisplacement curve provides a rich source of information allowing also for identification of non-linear events such as phase transformations, cracking or delamination of films [3].

Shape memory alloys (SMAs) are intermetallic materials exhibiting solid-to-solid phase transformation which is reversible. The phase change between austenite and martensite may be induced by temperature changes and/or deformation. If the material is deformed at a temperature higher than a certain limit $\left(A_{f}\right)$, it recovers original shape after unloading even if deformation reaches several percent; this effect is called superelasticity (SE). If deformed under this temperature, the deformation is mostly retained after unloading, which is called pseudo-

*corresponding author; e-mail: mfrost@it.cas.cz plasticity (PP), and the alloy must be heated over $A_{f}$ to fully recover its original shape. The different behavior is due to different phase composition. Above $A_{f}$ the only stable phase of material is austenite and martensite is stable only due to applied mechanical loading. With decreasing temperature, martensite becomes thermodynamically preferred. Martensitic phase features several (non-elastic) structural mechanisms which allow for recoverable accommodation of imposed strains; they are collectively termed reorientation (processes) hereinafter. As common in metals, however, if the mechanical loading is too severe, processes linked with plasticity are initiated and the original shape is fully recovered in neither situation.

Having found application in medicine, civil engineering or aerospace industry, NiTi-based alloys are a prominent class within SMAs usually utilized in form of a pollycrystalline bulk or film. Some material characteristics related to the phase transformation are quite sensitive to alloy composition, manufacturing and processing of final or semi-finished (wires, plates, films) products, e.g. [4, 5]. For instance, changes of composition and processing influence presence/absence of the intermediate phase, socalled $R$-phase, in NiTi alloys [6].

At least since the last decade, NiTi-based SMAs are intensively investigated also by means of (nano-) indentation techniques. For instance, it was observed in [7] that indentation induced $\mathrm{SE}$ is much more pronounced 
under spherical indentation conditions than that under pyramidal indentation. The quantitative stress analysis of nanoindentation on NiTi samples performed in [8] suggests that the stress required to induce the martensitic phase transformation at nanometer scales is on a par with macroscopic stresses required to induce it. Purely SE behavior (without any induced plasticity) was successfully observed in spherical indentation tests performed in [9]. Recently, work [10] studied dependence of nanoindentation behavior on the crystallographic loading direction on a coarse-grained NiTi sample. However, as pointed up by the authors, due to mutual interaction of microstructural processes in SMA, the mere load-displacement data are insufficient when it comes to identification of individual contributions to the net response. One option is to employ additional experimental investigations, the other is to rely on computational modeling.

There is an abundance of constitutive models of SMAs in the literature, originating at different scales of description and simplification, see reviews $[11,12]$ for further details. Recently, Sedlák et. al. developed a threedimensional constitutive model tailored for NiTi-based SMA exhibiting martensitic transformation via the intermediate $R$-phase [13]. So far, the model was extensively tested on simulations of thermomechanical response of products manufactured from NiTi SMA wires [14, 15]. Excellent correspondence between simulations and experiments has confirmed suitability of the underlying physical description of material processes and the apt choice of material parameters used as input for the model. However, indentation tests represent a very specific type of mechanical loading. Applicability of the model on simulations of these tests and sensitivity to a variation of the parameters should be explored prior to a routine use.

Hence, the aims of this contribution may be formulated as follows: (1) to prove functionality of the model in case of simulations of indentation tests performed on NiTi SMAs and to assess its present capabilities with respect to the experimental data, (2) to study differences in "idealized" material response at various temperatures by conducting a series of "virtual indentation tests", (3) to investigate how the occurrence of $R$-phase influences the simulations.

\section{Materials and experiments}

NiTi plate with composition 52.2 at.\% Ti, 47.8 at.\% Ni received from Johnson Matties Company were subjected to two types of indentation tests at room temperature. Samples were made by molding the plate into epoxy holder and mechanically polishing and finishing with $50 \mathrm{~nm} \mathrm{Al} \mathrm{Al}_{2} \mathrm{O}_{3}$ suspension to the final roughness of $10 \mathrm{~nm}$ determined by the atomic force microscopy (AFM).

Spherical nanoindentation was performed on Hysitron $\mathrm{P} / \mathrm{N}$ : TI-0043 indenter at room temperature, whereas CSM Ultra Nanoindentation Tester with Berkovich tip was utilized in a cyclic indentation test with the maximum force of $2 \mathrm{mN}$

\section{Constitutive model}

Numerical simulations within this work have been performed by own-developed User MATerial subroutine in the numerical software tool Abaqus. It is based on the recent constitutive model for NiTi-based SMAs formulated within the thermomechanical framework of Generalized Standard Materials [16]. It allows effective description for situations in which martensite forms from austenite through intermediate $R$-phase. Two main characteristic features of $R$-phase from the viewpoint of the model are its distinctly low shear modulus and the entropy change connected with the austenite- $R$-phase transition.

For description of a thermodynamic state of the material, two external state variables - total strain, $\varepsilon$, and temperature, $T$ - are complemented by two scalar internal variables - volume fraction of martensite, $\xi$, volume fraction of $R$-phase, $\eta$ and one tensorial internal variable - traceless inelastic strain related to transformation, $\varepsilon^{\mathrm{in}}$. The Helmholtz free energy is formulated in a rather standard form [11]:

$$
\begin{aligned}
& f\left(T, \varepsilon, \varepsilon^{\mathrm{in}}, \eta\right)=\frac{1}{2} K(\xi, \eta) \operatorname{tr}(\varepsilon)^{2} \\
& \quad+G(\xi, \eta)\left\|\operatorname{dev}(\varepsilon)-\varepsilon^{\mathrm{in}}\right\|^{2}+\Delta s^{\mathrm{AM}}\left(T-T_{0}\right) \xi \\
& \quad+\Delta s^{\mathrm{AR}}\left(T-R_{s}-\frac{\eta}{1-\xi} \frac{R_{f}-R_{s}}{2}\right) \eta+Q(T),
\end{aligned}
$$

where $Q(T)$ is a term depending on temperature only, hence not relevant under isothermal conditions studied in this work. Parameter $K$ represents bulk modulus of the material and, with respect to results in [17], it is supposed to be independent of phase composition. $G(\xi, \eta)$ represents the net shear modulus and it is determined from shear moduli of respective phases by the inverse rule of mixtures also known as the Reuss model. $\Delta s^{\mathrm{AM}}$ and $\Delta s^{\mathrm{AR}}$ denote the (positive) change of entropy related to the transitions austenite-martensite and austenite$R$-phase, respectively. $T_{0}$ is a material parameter related to thermodynamic equilibrium between austenite and martensite, whereas $R_{s}$ and $R_{f}$ are temperatures adjusting initiation and termination of transformation between austenite and $R$-phase under stress-free condition. More details on assumptions leading to this form can be found in [13]. The rate-independent dissipation function, which is directly related to so-called "transformation domain" common in phenomenological plasticity-inspired SMA models, takes the following form:

$$
d\left(T, \varepsilon^{\text {in }}, \xi, \dot{\varepsilon}^{\text {in }}, \dot{\xi}\right)=\left\{\begin{array}{c}
\Delta s^{\mathrm{AM}}\left[T_{0}-M_{s}+\xi\left(M_{s}-M_{f}\right)\right] \dot{\xi} \\
+\sigma^{\mathrm{reo}}(T)\left\|\dot{\varepsilon}^{\text {in }}\right\|, \quad \text { if } \dot{\xi} \geq 0, \\
\Delta s^{\mathrm{AM}}\left[T_{0}-A_{f}+\xi\left(A_{f}-A_{s}\right)\right] \dot{\xi} \\
+\sigma^{\mathrm{reo}}(T)\left[\left\|\frac{\dot{\xi}}{\xi} \varepsilon^{\text {in }}\right\|\right. \\
+\| \dot{\varepsilon}^{\text {in }}-\frac{\dot{\xi}}{\xi} \varepsilon^{\text {in } \|],} \quad \text { if } \dot{\xi}<0 .
\end{array}\right.
$$

Here, $A_{s}, A_{f}, M_{s}, M_{f}$ and $\sigma^{\text {reo }}(T)$ are material parameters influencing the hysteretic behavior of the model. 
The model incorporates the natural constraints on volume fractions of phases, i.e. they are restrained within interval $[0,1]$. Moreover, through a convex bound, $\left\langle\varepsilon^{\text {in }}\right\rangle \leq k \xi$, dependence of the maximal strain due to transformation (to martensite) on the applied loading mode is captured; the bounding function having the form

$$
\begin{aligned}
\left\langle\varepsilon^{\text {in }}\right\rangle & =\frac{I_{2}\left(\boldsymbol{D} \varepsilon^{\text {in }}\right)}{k} \\
& \times \frac{\cos \left(\frac{1}{3} \arccos \left(1-a\left[I_{3}\left(\boldsymbol{D} \varepsilon^{\text {in }}\right)+1\right]\right)\right)}{\cos \left(\frac{1}{3} \arccos (1-2 a)\right)}
\end{aligned}
$$

where $I_{2}(\boldsymbol{x})=\sqrt{\frac{2}{3} \boldsymbol{x}: \boldsymbol{x}}$ and $I_{3}(\boldsymbol{x})=4 \frac{\operatorname{det}(\boldsymbol{x})}{\left[I_{2}(\boldsymbol{x})\right]^{3}}$ for a tensor $\boldsymbol{x}$. Here $k$ is a material parameter representing the maximum transformation strain which can be attained in pure tension. Parameter $a$ captures the experimentally observed reduction of $k$ when pure compression is applied (the phenomenon is known as tension-compression asymmetry in the literature, see $[18,19]$ for details). Matrix $\boldsymbol{D}$ allows for the refined description of transformation strain anisotropy (e.g. because of texture) if experimental data are available. Since it is not the case of the present study, $D$ equals the unitary matrix hereinafter.

Based on prescribed strain and temperature, values of internal variables are obtained as the solution of a constrained minimization problem, in which sum of the free energy and the dissipation function is minimized with respect to the internal variables. Because of improved performance of the model, the inequality constraints are "smoothened" (through parameters $E^{\text {int }}$ and $c^{\text {reg }}$ ) when the solution is searched computationally. For more details on numerical treatment and implementation of the model to the finite element software package Abaqus see $[13,20]$.

\section{Numerical simulations}

Profiting from the axial symmetry of spherical indentation, the situation was modeled as a $2 \mathrm{D}$ axisymmetric problem using 6400 four-node linear axisymmetric elements (coded as CAX4 in Abaqus) in a square mesh whose lateral size represented $2400 \mathrm{~nm}$. Hence, the size of the sample is almost 40 times larger than the maximum indentation depth applied in this study $(65 \mathrm{~nm})$. The size of elements was spatially variable, decreasing in a geometrical sequence towards the corner directly under the tip, so that the mesh was most dense there, see Fig. 1a. Roller boundary condition was imposed on the axis of symmetry of the sample, bottom line was constrained in both radial and axial directions. This setting is common in such a type of simulations [21, 22]. Preliminary mesh sensitivity analysis was also performed to ensure the results are basically insensitive to the mesh size. Due to more than order of magnitude higher stiffness of the diamond indenter in comparison to the investigated alloy, the tip can be plausibly modeled as a rigid body [22-24], in the present situation a sphere with the a)

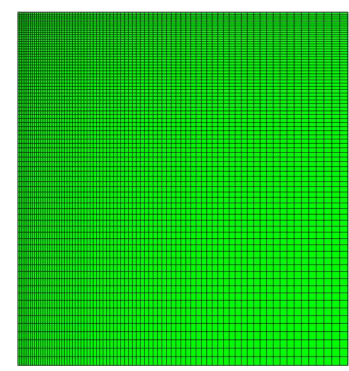

c)
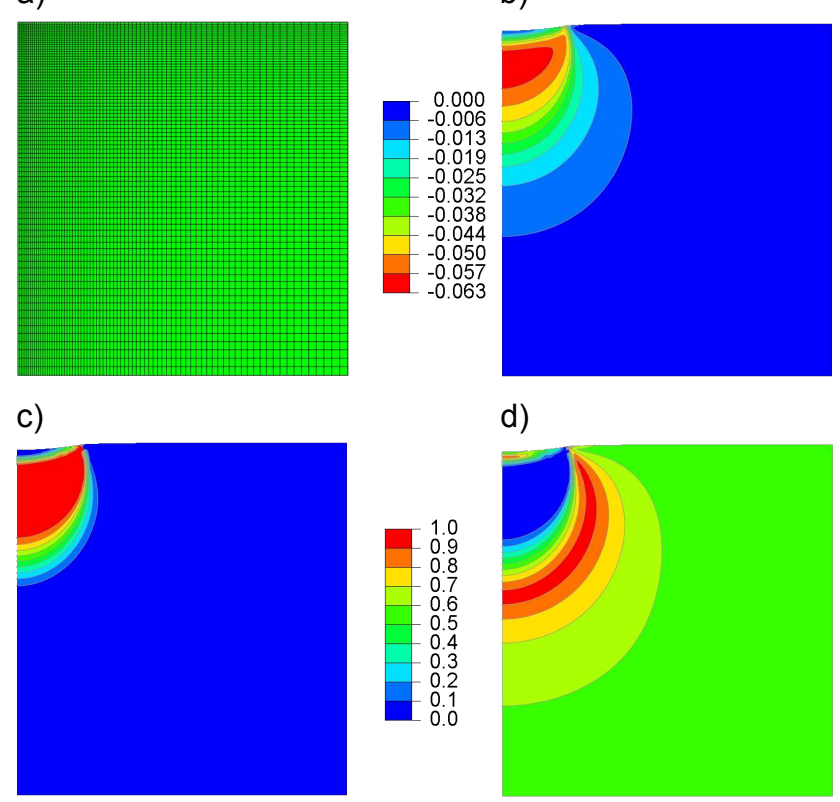

d)

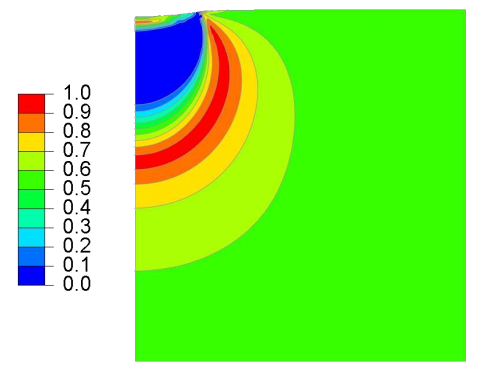

Fig. 1. a) finite element model of the sample, b) distribution of minimum of principal component of logarithmic strain, c) volume fraction of martensite, and d) volume fraction of $\mathrm{R}$-phase for spherical indentation at $42 \mathrm{~nm}$ depth and $0^{\circ} \mathrm{C}$.

radius of $2700 \mathrm{~nm}$. Moreover, due to the very low tangential angle between the sample and the spherical tip (up to $10^{\circ}$ and $13^{\circ}$ for indentation depths up to $42 \mathrm{~nm}$ and $65 \mathrm{~nm}$, respectively), the contact between the indenter and the mesh can be assumed frictionless [23]. Large rotations of material elements are dealt with by activation of the non-linear geometry mode in Abaqus software [25].

TABLE

Parameters of the constitutive model used in the simulations.

\begin{tabular}{c|c}
\hline \hline$K=150 \mathrm{GPa}$ & $E^{\mathrm{A}}=71 \mathrm{GPa}$ \\
\hline$E^{M}=71 \mathrm{GPa}$ & $E^{R}=42 \mathrm{GPa}$ \\
\hline$M_{s}=-42^{\circ} \mathrm{C}$ & $M_{f}=-44^{\circ} \mathrm{C}$ \\
\hline$A_{s}=-39^{\circ} \mathrm{C}$ & $A_{f}=-33^{\circ} \mathrm{C}$ \\
\hline$R_{s}=19^{\circ} \mathrm{C}$ & $R_{f}=-15^{\circ} \mathrm{C}$ \\
\hline$T_{0}=-40{ }^{\circ} \mathrm{C}$ & $c^{\mathrm{reg}}=0.01 \mathrm{MPa}$ \\
\hline$E^{\text {int }}=30 \mathrm{MPa}$ & $\Delta s^{\mathrm{AM}}=0.364 \mathrm{MPa} /{ }^{\circ} \mathrm{C}$ \\
\hline$\Delta s^{\mathrm{AR}}=0.121 \mathrm{MPa} /{ }^{\circ} \mathrm{C}$ & $k=0.065$ \\
\hline$a=0.9$ & $\sigma_{0}^{\mathrm{reo}}=160 \mathrm{MPa}$ \\
\hline$\Sigma^{\mathrm{reo}}=-0.9 \mathrm{MPa} /{ }^{\circ} \mathrm{C}$ &
\end{tabular}

Values of material parameters used in present simulations are summarized in the Table. Elastic properties as well as parameters $A_{s}, A_{f}, M_{s}, M_{f}, R_{s}, R_{f}$ were adjusted based on preliminary measurements of temperature dependence of elastic properties on temperature on $\mathrm{NiTi}$ samples by resonant ultrasound spectroscopy. Moreover, the Young modulus of martensite corresponds well to 


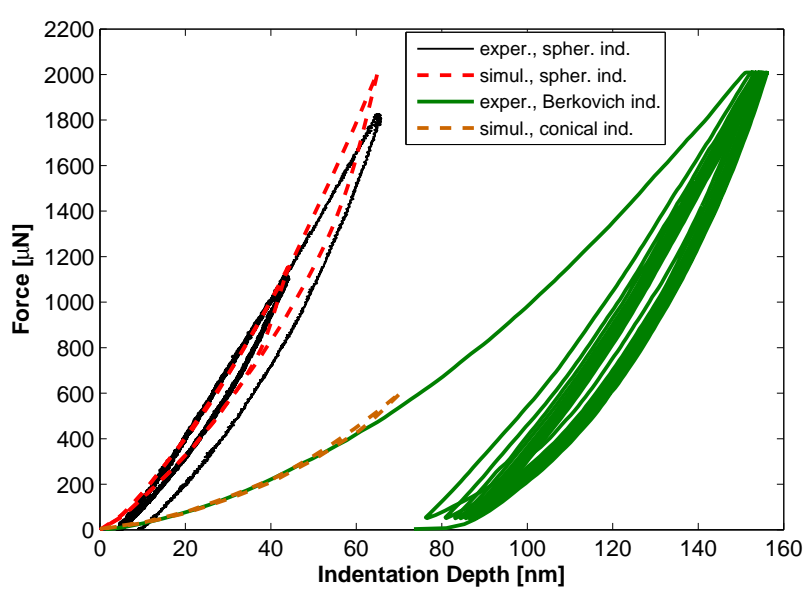

Fig. 2. Comparison of measured and simulated forceindentation depth dependence for indentation with spherical tip (left side) and Berkovich tip (right side) at $25^{\circ} \mathrm{C}$.

values determined from performed Berkovich indentation test using the Oliver-Pharr method. Values of parameters related to entropy change and to reorientation function $\sigma^{\text {reo }}(T)$ as well as numerical parameters $E^{\text {int }}$ and $c^{\text {reg }}$ were adopted from previous studies $[14,15]$. Parameters $a$ and $k$ strongly influence simulations, but their experimental analogies may substantially depend on manufacturing and processing of NiTi SMA products $[4,18]$. Their values were thus determined by fitting the model to available experimental data, see Fig. 2. Let us note that the fitted values match well with data commonly presented in the literature [26].

The left part of Fig. 2 presents the best fit of two simulations of spherical indentation together with experimental data. As can be seen from the measurements, indentation to maximum depth of $42 \mathrm{~nm}$ clearly exhibit SE behavior with zero residual depth, whereas indentation to $65 \mathrm{~nm}$ is not fully recovered after unloading. This indicates the initiation of irreversible processes in the material, most likely plasticity. They seem to be initiated during loading after the penetration depth exceeds $50 \mathrm{~nm}$, where the experimental response in loading departs from values predicted by simulation. Although the residual depth is only $\approx 1 / 6$ of the maximum indentation depth, it cannot be reflected in the simulation since the used constitutive model does not capture plasticity. For this reason, further simulations of spherical indentation will be conducted with indentation depths confined to $42 \mathrm{~nm}$.

Just for illustrative purposes, in the same figure, experimental data measured by the Berkovich indenter are compared to a simulation in which the pyramidal tip was approximated by a cone with equivalent half-angle $70.3^{\circ}[22,27,28]$; material data are the same. Its worth noting that the simulation again corresponds well to measurement in the initial part of loading which further validates the choice of material parameters and that the character of the experimental cyclic loading/unloading response confirms reversible transformation in the material [9]. One can conclude that the present model is able to realistically reproduce experimental indentation tests performed on NiTi SMA if irreversible processes are not involved.

Figure 3 presents a series of simulations performed at various temperatures with the same material data. As clearly seen, the force-indentation depth response strongly depends on temperature both in qualitative and quantitative sense. With decreasing temperature load needed to reach a given penetration depth decreases while the total dissipated energy manifested by the area of hysteresis increases. Moreover, for the lowest temperatures, a non-zero residual depth appears and its magnitude increases.

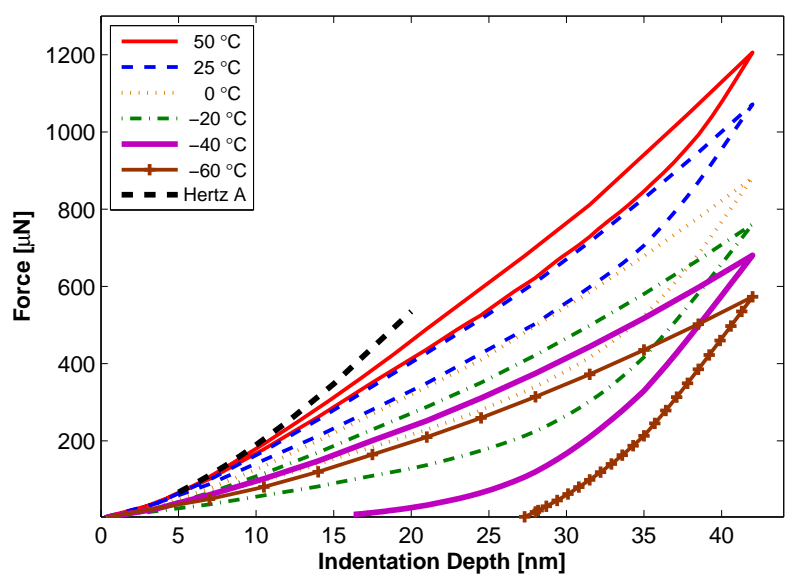

Fig. 3. Force-indentation depth dependence for a series of simulations of spherical indentation at various temperatures. The leftmost dashed line shows the corresponding analytical Hertzian solution for the purely elastic medium with elastic properties of austenite.

These phenomena are obviously related to phase transitions. For temperatures above $19^{\circ} \mathrm{C}$, sample is fully in austenite before loading and transition to R-phase is initiated first. Comparison with the corresponding analytical Hertzian solution for the purely elastic medium with elastic properties of austenite (dashed line) suggests that the process is initiated before $10 \mathrm{~nm}$ indentation depth is reached. With progression of loading, material in the most loaded region under the tip transforms further to martensite. At $25^{\circ} \mathrm{C}$, martensite is formed at lower stresses (due to temperature-stress coupling) than at $50{ }^{\circ} \mathrm{C}$, hence, the hysteresis loop after unloading is more pronounced in that case, see the figure.

At $0{ }^{\circ} \mathrm{C}$, the sample is more than $50 \%$ composed from $R$-phase prior to loading. Figure $1 \mathrm{~b}-\mathrm{d}$ provides information on spatial distribution of the minimum (i.e. negative) principal component of strain and volume fractions of phases at the end of loading, i.e. at penetration depth $42 \mathrm{~nm}$. The most strained region is fully in martensite as expected. However, in the region in which the strain component reaches about 0.02 a "crescent" of full $R$-phase 
occurs, effectively "softening" the force-indentation depth response in Fig. 3.

Martensite is the stable phase after unloading only when the indentation test is performed below $-20^{\circ} \mathrm{C}$. Then, the residual depth is non-zero as observed in Fig. 3 and the residual compressive stress just beneath the indented surface of the sample reach values corresponding to reorientation stress (i.e. $\sigma^{\text {reo }}(T)$ ). At $-60^{\circ} \mathrm{C}$, mainly martensite reorientation is under way during loading; hence, the unloading part of the curve is predominantly elastic.

Finally, due to the coupling of stress and temperature in "driving force" for phase transformations, the load needed to reach the prescribed penetration depth gradually decreases with decreasing indentation temperature.

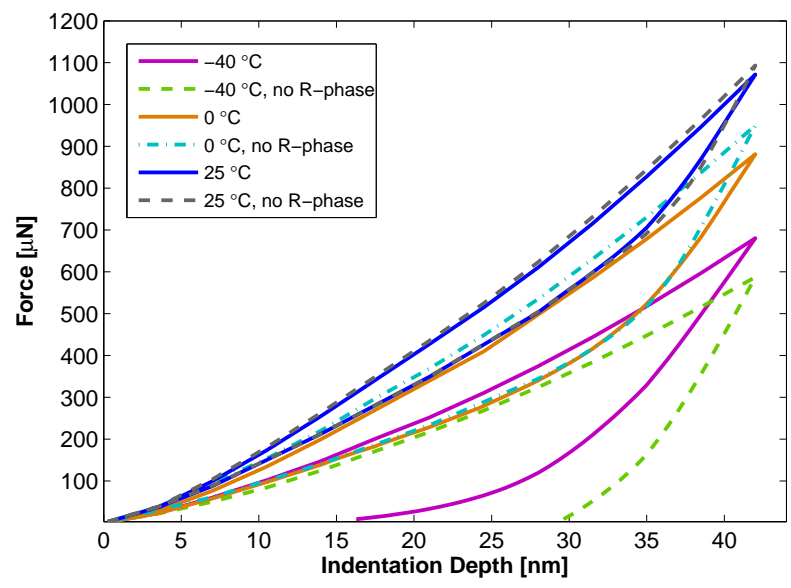

Fig. 4. Comparison of force-indentation depth dependence for direct martensite-austenite transformation and for the same transformation proceeding via intermediate R-phase.

The influence of $R$-phase to force-indentation depth curves can be investigated in Fig. 4. Three simulations from Fig. 3 are replotted there together with hypothetic response of the same material if transformation to $R$ phase would be suppressed (e.g. by different processing). To this end, we set $\Delta s^{\mathrm{AR}}=0$ and $G_{R}=G_{A}$ (i.e. $E_{R}=$ $\left.E_{A}\right)$ in the model. Differences are most pronounced at $-40^{\circ} \mathrm{C}$, where the residual depth is roughly twice higher. The reason is that if the $R$-phase transition is suppressed, more martensite (contributing by transformation strain to the residual depth) is formed in the sample at the same temperature. In the figure it is also worth to note different slopes of the initial part of the unloading curve related to the different elasticity of austenite and $R$-phase (best visible at $0^{\circ} \mathrm{C}$ ).

\section{Conclusions}

The recently developed constitutive model for NiTibased SMA including intermediate $R$-phase transformation was applied in simulations of indentation tests with the spherical tip. Employing experimental data the model was fitted to a particular sample material and a series of simulations mimicking indentation at various temperatures were performed. The results confirmed the good applicability of the utilized approach to such a kind of simulations and highlighted some features related to indentation of materials in which martensitic transformation proceed via intermediate $R$-phase. Simulations show that even in such case, spherical indentation to moderate depths can be used for a simple and fast detection whether the material is in superelastic or pseudoplastic regime. Viewed from the other side, "virtual experiments" allow for a qualified estimate of strain, stress and phase distribution before real experiments are performed; hence, they may provide guidelines for tailored experiments, e.g. avoiding initiation of irrecoverable processes. Extension of the constitutive model to capture irrecoverable processes is under development.

\section{Acknowledgments}

We would like to thank to Prof. T. Ben Zineb for possibility to perform computations on CLCM computation cluster (Centre Lorrain de calcul haute performance en mecanique, France). The research has been conducted within grant projects Nos. 14-28306P, 1415264S and 14-03044S of the Czech Science Foundation and supported by internal project No. M100761203 of the Academy of Sciences of the Czech Republic. The institutional support of the project RVO: 61388998 of IT ASCR, v.v.i. is also acknowledged.

\section{References}

[1] W.C. Oliver, G.M. Pharr, J. Mater. Res. 7, 1564 (1992).

[2] W.C. Oliver, G.M. Pharr, J. Mater. Res. 19, 3 (2004).

[3] A.C. Fischer-Cripps, Nanoindentation, 3rd ed., Springer, New York 2011.

[4] R. Delville, B. Malard, J. Pilch, P. Sittner, D. Schryvers, Acta Mater. 58, 4503 (2010).

[5] M. Kabla, H. Seiner, M. Musilova, M. Landa, D. Shilo, Acta Mater. 70, 79 (2014).

[6] K. Otsuka, X. Ren, Prog. Mater. Sci. 50, 511 (2005).

[7] W. Ni, Y.T. Cheng, D.S. Grummon, Appl. Phys. Lett. 82, 2811 (2003).

[8] C.P. Frick, T.W. Lang, K. Spark, K. Gall, Acta Mater. 54, 2223 (2006).

[9] M. Arciniegas, Y. Gaillard, J. Pena, J.M. Manero, F.J. Gil, Intermetallics 17, 784 (2009).

[10] J. Pfetzing-Micklich, Ch. Somsen, A. Dlouhy, Ch. Begau, A. Hartmaier, M.F.X. Wagner, G. Eggeler, Acta Mater. 61, 602 (2013).

[11] D.C. Lagoudas, P.B. Entchev, P. Popov, E. Patoor, L.C. Brinson, X. Gao, Mech. Mater. 38, 430 (2006).

[12] A. Khandelwal, V. Buravalla, Int. J. Struct. Changes 1, 111 (2009). 
[13] P. Sedlak, M. Frost, B. Benesova, P. Sittner, T. Ben Zineb, Int. J. Plast. 39, 132 (2012).

[14] M. Frost, P. Sedlak, A. Kruisova, M. Landa, J. Mater. Eng. Perform. 23, 2584 (2014).

[15] P. Sedlak, M. Frost, A. Kruisova, K. Hirmanova, L. Heller, P. Sittner, J. Mater. Eng. Perform. 23, 2591 (2014).

[16] Q.S. Nguyen, Stability and Nonlinear Solid Mechanics, Wiley, Chichester 2000.

[17] M.F.X. Wagner, W. Windl, Acta Mater. 56, 6232 (2008).

[18] Y. Liu, Z. Xie, J. Van Humbeeck, L. Delaey, Acta Mater. 46, 4325 (1998).

[19] K. Gall, H. Sehitoglu, Y.I. Chumlyakov, I.V. Kireeva, Acta Mater. 43, 1203 (1999).

[20] M. Frost, B. Benesova, P. Sedlak, Math. Mech. Solids, published online February 20, 2014.
[21] Q. Kan, W. Yan, G. Kang, Q. Sun, J. Mech. Phys. Solids 61, 2015 (2013).

[22] A.J. Muir Wood, T.W. Clyne, Acta Mater. 54, 5607 (2006).

[23] J.L. Bucaille, S. Stauss, E. Felder, J. Michler, Acta Mater. 51, 1663 (2003).

[24] W. Yan, Q. Sun, X.-Q. Feng, L. Qian, Int. J. Solids Struct. 44, 1 (2007).

[25] Abaqus Reference Manuals, SIMULIA Inc, USA 2010.

[26] K. Bhattacharya, R.V. Kohn, Acta Mater. 44, 529 (1996).

[27] M. Lichinchi, C. Lenardi, J. Haupt, R. Vitali, Thin Solid Films 312, 240 (1998).

[28] M. Dao, N. Chollacoop, K.J. van Vliet, T.A. Venkatesh, S. Suresh, Acta Mater. 49, 3899 (2001). 